\title{
The prognostic factors of stage IV colorectal cancer and assessment of proper treatment according to the patient's status
}

Hae Ran Yun • Woo Yong Lee • Won Suk Lee •

Yong Beom Cho • Seong Hyeon Yun • Ho-Kyung Chun

Published online: 7 July 2007

(C) Springer-Verlag 2007

The given name of the third author was rendered incorrectly. The correct spelling is Won Suk Lee, as given here.

The name of the University School of Medicine in the first address was rendered incorrectly. The correct spelling is Sungkyunkwan, as given here.

The online version of the original article can be found at http://dx.doi. org/10.1007/s00384-007-0315-x.

H. R. Yun · W. Y. Lee $\cdot$ W. S. Lee $\cdot$ Y. B. Cho $\cdot$ S. H. Yun •

H.-K. Chun

Department of Surgery, Samsung Medical Center,

Sungkyunkwan University School of Medicine,

Seoul, South Korea

W. Y. Lee $(\bowtie)$

50 Il-One dong Kang-Nam gu,

Seoul, South Korea

e-mail: seeskywau@naver.com 